\title{
Modifying closed-book exams for use as open-book exams
} School of Mathematics, 30 March 2020

\author{
Toby Bailey, George Kinnear, Steven O'Hagan and Chris Sangwin. \\ Comments or corrections should be sent to Chris (C.J.Sangwin@ed.ac.uk).
}

In response to Covid-19, university mathematics courses will switch to remote teaching and replace traditional exams with alternatives. The School of Mathematics at the University of Edinburgh has agreed to use take home exams for courses in years 3 and later. In the summer of 2020, each exam will be an open-book version of the original exam, taken remotely and unsupervised. This has potentially serious consequences because the exam questions may take on a different character in open- versus closed-book settings, and because of concerns about possible academic misconduct.

This document outlines some ways that existing exam questions can be evaluated and modified to address these concerns. We hope this will provide colleagues with some practical suggestions for adjusting their exam papers, as well as showing students examples of how exam questions might look different. Small changes to existing exam papers should be sufficient; the aim should be to maintain the overall level of difficulty.

In the next section, we give some general principles to guide this work. Then, in the following section, we show some concrete examples of how questions could be modified, using questions taken from past exams at the University of Edinburgh.

\section{Principles}

1. Students still need to understand the subject to do well in an open-book exam. Experience of using open-book exams in pre-honours courses suggests that questions where the answer is "in the book" are not answered well by students who have not studied properly (unless perhaps the answer can be easily located in the book even if you have not studied it).

\section{Avoid extensive "bookwork".}

Traditional exams often feature "bookwork" e.g. quoting standard definitions, results, or proofs; or carrying out a standard calculation. When revising an existing exam, it is helpful to quantify the extent of such questions before making modifications. The aim is to avoid having answers which appear directly in course materials or on the web.

\section{Open-book exams are better suited to questions requiring higher-order skills.} Robert Talbert suggested that when writing mathematics examinations we could move up a level or two on Bloom's Taxonomy, i.e. favouring application, analysis, and synthesis rather than just remembering and understanding (Talbert, 2020). Lower level questions often have unique answers, making it hard to distinguish independent student work from work which has been plagiarised or has come from collusion with another student. Again, it would be helpful to quantify the marks available for such questions, before making modifications. How many marks are assigned to parts for which it will be difficult to distinguish between correct answers from different students?

The MATH taxonomy (Smith et al., 1996) is a helpful tool for thinking about different types of mathematics tasks. It is a mathematics-specific version of Bloom's taxonomy, with various types of question organised into the three groups shown in Table 1. The suggestion is to reduce the use of Group A questions in open-book exams. This will avoid questions where students may have access to model answers in lecture notes or online, or indeed may be 
able to use tools like WolframAlpha or other computer algebra systems. These issues are discussed, and other practical suggestions are made, in (Pointon \& Sangwin, 2003).

\begin{tabular}{|c|c|c|}
\hline Group & Category & Example question \\
\hline \multirow{3}{*}{$\begin{array}{l}\text { A } \\
\begin{array}{l}\text { Factual recall and routine } \\
\text { procedures }\end{array}\end{array}$} & $\begin{array}{l}\text { Factual knowledge } \\
\text { and fact systems }\end{array}$ & State the definition of orthogonal matrix. \\
\hline & Comprehension & $\begin{array}{l}\text { Which of the following matrices are } \\
\text { orthogonal? (a) }\left[\begin{array}{ll}4 & 0 \\
0 & \frac{1}{4}\end{array}\right] \text { (b) ... }\end{array}$ \\
\hline & $\begin{array}{l}\text { Routine use of } \\
\text { procedures }\end{array}$ & $\begin{array}{l}\text { Find the eigenvalues and corresponding } \\
\text { eigenvectors of the matrix }\left[\begin{array}{cc}2 & 0 \\
-1 & 3\end{array}\right]\end{array}$ \\
\hline \multirow{4}{*}{$\begin{array}{c}\text { B } \\
\text { Using existing mathematical } \\
\text { knowledge and techniques in } \\
\text { new ways }\end{array}$} & Information transfer & $\begin{array}{l}\text { For what value of } k \text { do the following vectors } \\
\text { NOT form a basis of } \mathbb{R}^{3} \text { ? }\end{array}$ \\
\hline & & $(-2)(16)(-3)$ \\
\hline & & $\left(\begin{array}{c}1 \\
-1\end{array}\right),\left(\begin{array}{c}-14 \\
k\end{array}\right),\left(\begin{array}{c}3 \\
-2\end{array}\right)$ \\
\hline & $\begin{array}{l}\text { Application in new } \\
\text { situations }\end{array}$ & $\begin{array}{l}\text { Given that }\left[\begin{array}{cc}1 & -2 \\
2 & 1\end{array}\right] \cdot A \cdot\left[\begin{array}{cc}1 & 2 \\
-2 & 1\end{array}\right]=5 \cdot I, \\
\text { evaluate the determinant of matrix } A .\end{array}$ \\
\hline \multirow{4}{*}{$\begin{array}{c}\text { C } \\
\text { Application of conceptual } \\
\text { knowledge to construct } \\
\text { mathematical arguments }\end{array}$} & $\begin{array}{l}\text { Justifying and } \\
\text { interpreting }\end{array}$ & $\begin{array}{l}\text { Describe the geometric effect of the } \\
\text { transformation } T: \mathbb{R}^{2} \rightarrow \mathbb{R}^{2} \text { given by }\end{array}$ \\
\hline & & $T(\boldsymbol{v})=\left[\begin{array}{cc}1 & -1 \\
1 & 1\end{array}\right] v$ \\
\hline & $\begin{array}{l}\text { Implications, } \\
\text { conjectures and } \\
\text { comparisons }\end{array}$ & $\begin{array}{l}\text { Give an example of a three-by-three matrix } \\
\text { with real entries (i.e. in } \mathbb{R}^{3 \times 3} \text { ) with exactly } \\
\text { one real eigenvalue which is non-zero, and } \\
\text { also with two complex eigenvalues. } \\
\text { Repeated eigenvalues will be counted with } \\
\text { multiplicity. }\end{array}$ \\
\hline & Evaluation & Not commonly observed. \\
\hline
\end{tabular}

Table 1. Overview of the MATH taxonomy, adapted from Darlington (2014) and Kinnear et al. (2020). The example questions shown are all based on the Year 1 course, Introduction to Linear Algebra.

\section{References}

Darlington, E. (2014). Contrasts in mathematical challenges in A-level Mathematics and Further Mathematics, and undergraduate mathematics examinations. Teaching Mathematics and lts Applications, 33(4), 213-229. https://doi.org/10.1093/teamat/hru021

Kinnear, G., Bennett, M., Binnie, R., Bolt, R. and Zheng, Y. (2020). "Reliable application of the MATH taxonomy sheds light on assessment practices". Teaching Mathematics and its Applications: An International Journal of the IMA. hrz017 URL: https://doi.org/10.1093/teamat/hrz017

Pointon, A., \& Sangwin, C. J. (2003). An analysis of undergraduate core material in the light of handheld computer algebra systems. International Journal of Mathematical Education in Science and Technology, 34(5), 671-686. https://doi.org/10.1080/0020739031000148930

Smith, G., Wood, L., Coupland, M., Stephenson, B., Crawford, K., \& Ball, G. (1996). Constructing mathematical examinations to assess a range of knowledge and skills. International Journal of Mathematical Education in Science and Technology, 27(1), 65-77. https://doi.org/10.1080/0020739960270109

Talbert, R. (2020). How to have students submit handwritten work in an online setting. Retrieved March 26, 2020, from https://rtalbert.org/handling-timed-exams-in-the-online-environment/amp/ 


\section{Practical suggestions}

1. Try using Google to find answers to questions; if an answer is easy to find (say within the first three pages of results) then consider modifying it.

\section{Example}

- Linear Analysis (1)(a)

2. Keeping questions unchanged but redistributing marks away from factual recall and routine procedures may be sufficient.

\section{Example}

- Honours Algebra (2)(d)

3. Removing steps from questions that are intended to lead students to a solution could make questions more suitable for open-book exams. However, it will likely also increase the question difficulty.

\section{Example}

- Linear Analysis (1)(a)

4. Questions about concrete (unfamiliar) examples are likely to be harder to Google than general results. Even "standard" examples may make for effective questions if you omit their names or change notation.

\section{Example}

- Linear Analysis (2)(b)

5. You may still be able to assess students' understanding of proof by asking proofcomprehension questions rather than asking for them to reproduce a proof.

\section{Examples}

- Honours Algebra (3)

- Theory of Statistical Inference (2)(b)(i)

\section{Examples}

The examples discussed are taken from past papers or mock exams. In each case, the current course lecturer identified questions that they felt were unsuitable for open-book exams; we discuss possible light-touch modifications to make them more suitable.

In each example, questions that might be unsuitable are highlighted in yellow. 


\section{Honours Algebra}

(2) (a) Let $\mathcal{S}(2)=\left(\vec{e}_{1}, \vec{e}_{2}\right)$ be the standard basis of $V=\mathbb{R}^{2}$ and let $\mathcal{B}=\left(\vec{v}_{1}=2 \vec{e}_{1}+\right.$ $\left.\vec{e}_{2}, \vec{v}_{2}=\vec{e}_{1}+2 \vec{e}_{2}\right)$. Show that $\mathcal{B}$ is a basis of $V$. Now suppose that a linear mapping $f: V \rightarrow V$ is represented with respect to $\mathcal{S}(2)$ by the matrix

$$
A=\left(\begin{array}{cc}
3 & 2 \\
-4 & 2
\end{array}\right)
$$

Find the matrix $B$ that represents $f$ with respect to $\mathcal{B}$. Write down an equation that expresses the relationship between $A$ and $B$.

[7 marks]

(b) Let $U \subseteq V$ be a subspace of a vector space. Define the quotient vector space $V / U$ including the definition of addition and scalar multiplication in $V / U$. Define the canonical mapping can : $V \rightarrow V / U$. What is the kernel of can? [6 marks]

(c) Let $Y \subseteq V / U$ be a subspace. Show that

$$
\operatorname{can}^{-1}(Y)=\{\vec{v} \in V: \operatorname{can}(\vec{v}) \in Y\}
$$

is a subspace of $V$ that contains $U$. Let $X \subseteq V$ be a subspace such that $U \subseteq X$. Show that

$$
\operatorname{can}(X)=\{\operatorname{can}(x): x \in X\}
$$

is a subspace of $V / U$.

[7 marks]

(d) State the Rank-Nullity Theorem carefully and clearly. Apply it to give formulas for the dimension of the above subspaces $\operatorname{can}(X)$ and $\operatorname{can}^{-1}(Y)$ in terms of the dimensions of $U, X$ and $Y$.

[5 marks]

(2) (b) There are 6 marks overall for this part but no specific mark allocation for stating the definition of a quotient vector space. The marking scheme could be modified so that few (or no) marks are awarded for simply stating the definition.

(2) (d) Could be reformulated as "Apply the Rank-Nullity Theorem to give formulas..." but then this gives students less direction to state the theorem carefully and how it applies - so perhaps actually leaving the question as-is would be better in this case. 
(3) Throughout this question $R$ will denote a ring.

(a) Define what is meant by saying $R$ is an integral domain. State the Cancellation Law for Integral Domains. [You do not need to prove it.] Prove that a finite integral domain is actually a field.

[7 marks]

(b) Describe all the units in the polynomial ring $R[X]$ where $R$ is an integral domain. Briefly justify your answer. Give a counterexample to your description in the case $R$ is not an integral domain.

[4 marks]

(c) Let $f: R \rightarrow S$ be a ring homomorphism. Define the kernel and image of $f$ and prove that the kernel is an ideal of $R$. State the First Isomorphism Theorem for Rings.

[7 marks]

(d) Let $\mathbb{F}_{2}$ be the field with 2 elements. Let $P=X^{3}+X^{2}+1 \in \mathbb{F}_{2}[X]$. Let $I$ be the ideal of $\mathbb{F}_{2}[X]$ generated by $P$. List the elements of the factor ring $S=\mathbb{F}_{2}[X] / I$. This list should include each element once and only once. Prove that $S$ is a field. Produce a commutative ring $T$ with the same number of elements as $S$, but that is not isomorphic to $S$ as a ring.

[7 marks]

(3) (a) This is entirely "bookwork", writing down a definition and a theorem, and giving a standard proof that appears in lecture notes. In addition, a web search for "finite integral domain is actually a field" will immediately bring up a relevant argument.

(3) (b) Searching "units in the polynomial ring over integral domain" brings up some relevant material.

(3) (c) Appears in lecture notes and can be easily found online.

One possibility is to change to a proof-comprehension question, i.e. presenting students with a proof and asking questions about it. For instance, for (a), the model solution is:

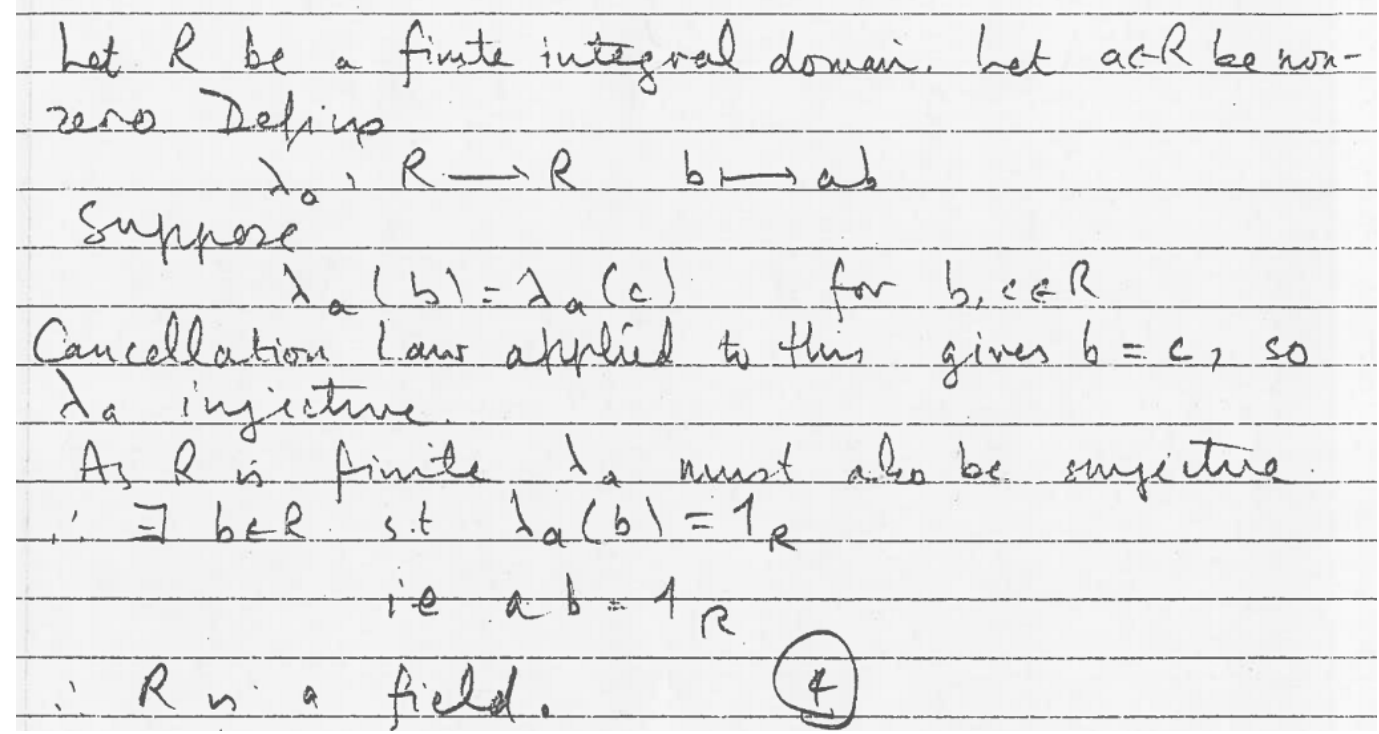

The exam question could include a version of this, and students could be asked to explain:

- Why is it valid to conclude that $R$ is a field in the final line?

- Why does the fact that $R$ is finite mean $\lambda_{a}$ is surjective? 
Linear Analysis

Based on comments from Jim Wright

(1) Let $(X,\|\cdot\|)$ be a normed linear space.

(a) For any $x, y \in X$, show that $|\|x\|-\|y\|| \leq\|x-y\|$.

[2 marks]

(b) Suppose that $x_{n} \rightarrow x$ in $(X,\|\cdot\|)$. Show that $\left\|x_{n}\right\| \rightarrow\|x\|$ as a sequence of real numbers.

[1 mark]

(2) Let $X$ be a vector space over the reals $\mathbb{R}$ and let $\|\cdot\|_{1}$ and $\|\cdot\|_{2}$ be two norms on $X$.

(a) Explain what it means to say that $\|\cdot\|_{1}$ and $\|\cdot\|_{2}$ are equivalent norms.

[2 marks]

(b) Suppose that $\|\cdot\|_{1}$ and $\|\cdot\|_{2}$ are equivalent norms on $X$. If a set $S \subset X$ is closed with respect to $\|\cdot\|_{1}$, show that it is closed with respect to $\|\cdot\|_{2}$. [3 marks]

(c) Now suppose that $X=C[0,1]$ is the space of real-valued, continuous functions on $[0,1]$. Recall the two norms

$$
\|f\|_{L^{1}}=\int_{0}^{1}|f(x)| d x \text { and }\|f\|_{L^{\infty}}=\sup _{x \in[0,1]}|f(x)|
$$

on $C[0,1]$.

(i) Show that $\|f\|_{L^{1}} \leq\|f\|_{L^{\infty}}$ for all $f \in C[0,1]$.

[1 mark]

(ii) Suppose that $M \subset C[0,1]$ is a closed subspace with respect to $\|\cdot\|_{L^{1}}$. Show that $M$ is closed with respect to $\|\cdot\|_{L^{\infty}}$.

[2 marks]

(iii) Consider the subspace $M=\{f \in C[0,1]: f(0)=0\}$.
A. Show that $M$ is closed with respect to $\|\cdot\|_{L^{\infty}}$.
[2 marks]
B. Show that $M$ is not closed with respect to $\|\cdot\|_{L^{1}}$.
[2 marks]
C. Show that the closure of $M$ in $\left(C[0,1],\|\cdot\|_{L^{1}}\right)$ is $C[0,1]$.
[2 marks]

(3) Let $(X,\|\cdot\|)$ be a Banach space and $M \subset X$ a closed subspace of $X$. Show that $(M,\|\cdot\|)$ is a Banach space.

[3 marks]

(1) (a) This result was quoted and used many times in the course. A proof was never presented but it is quite easy to find proofs of it online. A search for "normed linear space triangle inequality" and a couple of clicks leads to:

https://en.wikipedia.org/wiki/Triangle inequality which has a proof.

Part (a) could be removed and the number of marks awarded for (b) increased. Without the prompt to use the "reverse triangle inequality", this question could be quite a bit harder as students would have to select a strategy first. Stating the reverse triangle inequality in the question and telling students that they may use it without proof is another option.

(2) (a)

(3) These can easily be looked up in lecture notes.

(2) (b) Can be replaced by (2)(c)(ii) which is a part realisation of the general fact (2)(b) for a particular example. 


\section{Based on comments from Tim Cannings}

1. $\mathbf{y}=\left\{y_{1}, \ldots, y_{n}\right\}$ are observations of random variables $Y_{1}, \ldots, Y_{n}$ that are independent and have an exponential distribution with parameter $\lambda$.

(a) Find a minimal sufficient statistic for $\lambda$.

[2 marks]

(b) Determine the moment generating function, $\mathbb{E}\left(\mathrm{e}^{t Y}\right)$, for the exponential distribution and show that the moment generating function of the $\Gamma(a, b)$ distribution is

$$
\mathbb{E}\left(\mathrm{e}^{t Y}\right)=\left(\frac{b}{b-t}\right)^{a}
$$

Hence, or otherwise, find the distribution of the minimal sufficient statistic obtained in (a). Hint: the probability density function for $\Gamma(a, b)$ is $f(x ; a, b)=b^{a} x^{a-1} \mathrm{e}^{-b x} / \Gamma(a)$.

[5 marks]

(c) Show that $\hat{\theta}=Y_{1}$ is an unbiased estimator of $\theta=1 / \lambda$ and find its variance. [3 marks]

(d) State the Rao-Blackwell theorem. Use it to obtain an estimator of $\theta$ with smaller variance. Find the variance of the new estimator.

[6 marks]

(e) Show that the new estimator is unbiased, efficient and consistent.

[6 marks]

(f) Show that the estimator obtained in (d) coincides with the maximum likelihood estimator for $\theta$.

[3 marks]

(1) (d) Stating the theorem becomes straightforward. However, no marks are allocated to stating the theorem, only the part of the question overall. So this question could remain unchanged but with little or no marks awarded for simply stating the theorem. 
2. Let $y_{1}, \ldots, y_{n}$ be observations of random variables $Y_{1}, \ldots, Y_{n}$ that are independent and drawn from the Pareto distribution with density

$$
f(y ; \theta, \alpha)=\left\{\begin{array}{ll}
0 & y<\theta \\
\frac{\alpha \theta^{\alpha}}{y^{\alpha+1}} & y \geq \theta
\end{array} .\right.
$$

(a) Find a minimal sufficient statistic for $(\alpha, \theta)$.

(b) Suppose now that $\theta$ is fixed and known.

(i) Is the resulting distribution in the exponential family?

(ii) State and prove the Cramér-Rao bound on the variance of an estimator of a parameter $\lambda$.

(iii) Obtain a lower bound on the variance of an unbiased estimator of $\alpha$. Is this bound achievable? If so, give an estimator that achieves the bound.

[3 marks]

(iv) Suppose now that we are interested in biased estimators of $\alpha$. Is the Cramér-Rao bound achievable for biased estimators? If so, find an estimator that achieves the bound and explicitly compute the bias of that estimator.

[5 marks]

(c) Now assume that $\alpha$ is fixed and known, with $\alpha>2$.

(i) Is this distribution in the exponential family?

[1 mark]

(ii) Find the distribution of $Y_{(1)}$, the minimum of the $Y_{i}$ 's. Hence, or otherwise, find an unbiased estimator of $\theta, \hat{\theta}$.

[3 marks]

(iii) Compute the Cramér-Rao bound on the variance of unbiased estimators of $\theta$. Does the bound apply in this case?

(iv) Find the variance of the estimator $\hat{\theta}$. Comment on your answer.

[3 marks]

2. (b) (ii) The statement and proof could be copied without understanding.

For a long proof like this, it may make sense to give the proof (or part of it) to the students, with some key steps numbered, and ask them to justify the steps fully or explain why conditions are necessary.

It may also be possible to ask, for example: "Write a paragraph outlining the main steps of the proof of the Cramer-Rao bound on the variance of an unbiased estimator. Indicate where each assumption made in the result is used." 